\title{
An Improved High-Gain Amplifier Circuit Model Used in UHF RFID Receiver
}

\author{
X.Y. Hao, X.H. Yan \\ College of electronic science \& engineering \\ Nanjing University of Posts and Telecommunications \\ Nanjing Peoples Republic of China
}

\begin{abstract}
This paper presents an improved high-gain amplifier circuit with low-frequency servo loop to remove DC offset and 1/f noise in UHF RFID receiver. Compared to AC coupling amplifier, the circuits can overcome DC saturation and reduce the distortion of the baseband signal in various data rate. Based on the UHF RFID data rate as example, the paper gives the circuit design model and detailed parameters, and the simulation and practical experiments are carried out to validate the circuit model.
\end{abstract}

\section{Keywords-UHF; RFID; amplifier; DC offset; servo loop}

\section{INTRODUCTION}

UHF RFID system is a direct conversion receiver (DCR), the RF signal is converted directly to the baseband signal, the frequencies of baseband signal start from zero. In the receiver, in order to maintain the integrity of the baseband signal, the full bandwidth DC amplifier is the preferred option. However, the presence of DC offset and carrier leakage in circulator [Figure 1] will saturate the following amplifier, and the 1/f noise will destroy signal integrity [1][2][3].

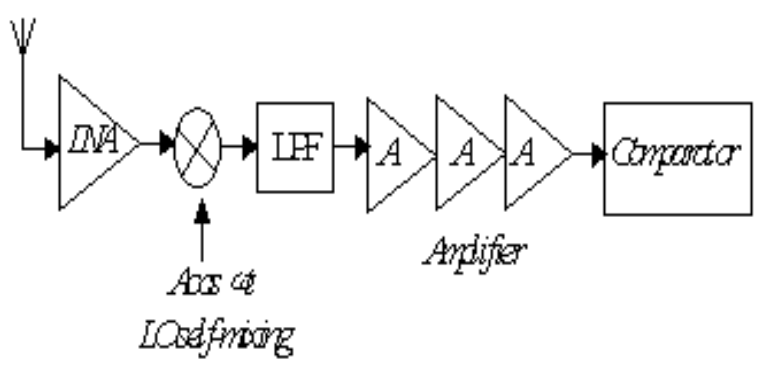

\section{FIGURE I. .THREE MAIN SOURCES OF LEAKAGE GIVING RISE TO DC OFFSET IN UHF RFID. (1) LO SELF-MIXING, (2) SELF- MIXING INTERFERER, (3) DC DUE TO MISS-MATCH OF AMPLIFIER.}

Therefore the AC coupling circuit is used to solve the problem, for a given signal transmission rate, the $\mathrm{AC}$ coupling mode is simple and effective, but the transmission data rate changes from $16 \mathrm{kbps}$ to $320 \mathrm{kbps}$ according to the new standard of ISO $18000-6 \mathrm{C}$, the fixed constant ACcoupled amplifier is hard to adapt to various data rate

The simulation is shown in Figure 2, for the low data rate transmission, the signal wave will produce differential effects, and for the high data rate transmission, because of response effect of capacitance, the signal will produce inter-symbol interference phenomenon.

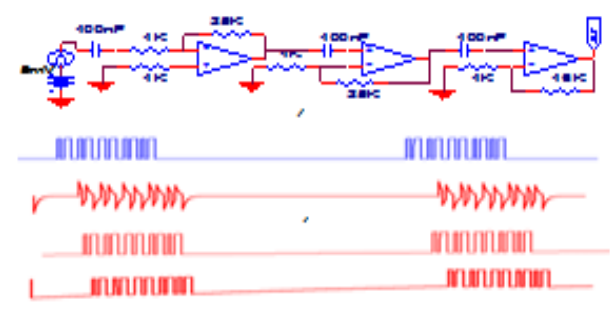

FIGURE II. (A) AC COUPLING AMPLIFIER WITH 100DB GAIN, (B) INPUT MANCHESTER CODE SIGNAL, (C)(D) And (E)Indicate Output Wave In Low, Middle And High Data Rate.

Some sophisticated equalization algorithm is used to solve signal integrity issues. However, practice shows that it's difficult to achieve high performance only relying on algorithms to correct the distorted signal. Especially in the process of multi-tag reading, the signal quality seriously impact on the efficiency of anti-collision algorithm.

In addition to using $\mathrm{AC}$ coupling methods, other methods are proposed to solve DC offsets, in [4] [5] a FIR equalizer or HPF filter are introduced to mitigate the effects in a DCR, in [6] an averaging circuit is introduced to remove the DC offsets by subtracting the estimated DC value. Such methods rely on A/D converter and corresponding software method to compensate the defects, yet the compensation lag will affect system performance and increase the complexity of the system.

In this paper, a new novel wide-band and high gain amplifier with low frequency servo loop is designed, The experimental results show that, this circuit can keep the integrity of the baseband signal without the aid of software algorithm, and it can adapt to any data transmission speed in UHF RFID system, it is shown in Figure 3.

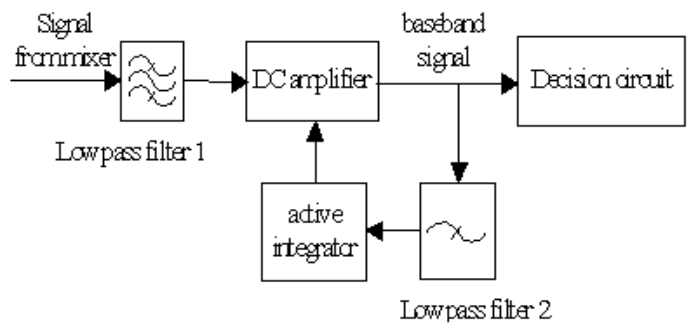

FIGURE III. DC AMPLIFIER CIRCUIT WITH LOW-FREQUENCY SERVO LOOP. 


\section{THE PRINCIPLE OF THE IMPROVED DC AMPLIFIER CIRCUIT}

The circuits contain two low pass filters, a multi-stage wideband DC amplifier, an integrator and an active threshold decision circuit. The high-frequency signal of mixer is filtered by the low-pass filter 1 and baseband and DC signal are retained. The multi-stage DC amplifiers have large dynamic scope, the gain is set $100 \mathrm{db}$ in the simulation circuit, and the full-power bandwidth of amplifier satisfies the maximum rate requirements of UHF RFID. The low-pass filter 2 and the Integrator circuit are the core component to cancel the effect of DC and 1/f noise, the circuits filter out the AC component from the DC amplifier while retaining its DC and low frequency component, the active integrator accumulate the voltage and feed back to the invert input of the high-speed DC amplifier, because the integrator samples the signal from the last amplifier, no matter how much DC component produced by the front mixer or by the high-speed operational amplifier self, the final DC offset voltage is limited to a very small level. Based on the above circuit principle, in order to remove the $1 / \mathrm{f}$ noise of baseband signal, the low pass filter 2 and integrator must be set a higher cut off frequency, but in order to try to maintain the integrity of the baseband signal, the cut off frequency should be set lower.

The baseband signal is series rectangle pulse, according to Fourier transform, the rectangular pulse can be expressed

$$
\begin{aligned}
& \left|\mathrm{F}_{\mathrm{n}}\right|=\frac{1}{2} \sqrt{a_{n}^{2}+b_{n}^{2}}=\frac{1}{2} \sqrt{\frac{4 \sin ^{2}\left(\frac{n \omega_{0} \tau}{2}\right)}{n^{2} \pi^{2}}} \\
& =\frac{1}{2} \cdot \frac{2 \sin \left(\frac{n \omega_{0} \tau}{2}\right)}{n \pi}=\frac{\sin \left(\frac{n \omega_{0} \tau}{2}\right)}{n \pi}
\end{aligned}
$$

Power spectrum is expressed

$$
\begin{gathered}
\varphi(\omega)=2 \pi \cdot \sum_{n=-\infty}^{\infty} \frac{\sin ^{2}\left(\frac{n \omega_{0} \tau}{2}\right)}{n^{2} \pi^{2}} \delta\left(\omega-n \omega_{0}\right) \\
=2 \pi \sum_{n=-\infty}^{\infty} \frac{\sin ^{2}\left(n \pi \cdot \frac{\tau}{T}\right)}{n^{2} \pi^{2}} \delta\left(\omega-n \omega_{0}\right)
\end{gathered}
$$

The average normalized power spectral density of a series of rectangular pulses representing $\mathrm{n}$ data bits is thus

$$
G(f)=\frac{n A^{2} \tau^{2} \sin c^{2}(\pi f \tau)}{n \tau}=A^{2} \tau \sin c^{2}(\pi f \tau) \text { volts }{ }^{2} / H z
$$

Generally, the percentage of signal's power within a frequency band is a good indication of the signal distortion. According to the standard of ISO $18000-6 \mathrm{C}, 15 \%$ deviation of the receiver pulse is permitted [7]. By the circuit simulation result, it is appropriate that the cut off frequency is set $0.1 \%$ of the Manchester data clock, the minimum data speed of the ISO $18000-6 \mathrm{C}$ standard is $16 \mathrm{kbps}$, and the Manchester code clock is $32 \mathrm{kHz}$, so the cut off frequency of low pass filter 2 can be set $32 \mathrm{~Hz}$.

At the same time, the cut off frequency determines the offset $1 / \mathrm{f}$ noise, according to the typical MOSFET amplifiers model, the $1 / \mathrm{f}$ noise in the vicinity of $1 \mathrm{KHz}$ can be expressed

$$
\left.\frac{K}{W L C_{O X}} \cdot \frac{1}{f}\right|_{f \approx 1 \mathrm{KHz}} \approx 4 k T \frac{2}{3 g_{m}}
$$

The $1 / \mathrm{f}$ noise power in a bandwidth from $0 \mathrm{~Hz}$ to $32 \mathrm{~Hz}$ can be calculated,

$$
\begin{aligned}
& P_{n 1}=\int_{O H z}^{32 H z} \frac{K}{W L C_{O X}} \cdot \frac{d f}{f} \\
& =\frac{K}{W L C_{O X}} \ln 32 \\
& =4 k T \frac{2}{3 g_{m}}(1 \mathrm{KHz}) \ln 32
\end{aligned}
$$

The thermal noise, we have

$$
\begin{aligned}
& P_{n 2}=\int_{0 H z}^{32 H z} \frac{K}{W L C_{O X}} \cdot d f \\
& =\int_{0 H z}^{32 H z} 4 k T \frac{2}{3 g_{m}} d f \\
& =4 k T \frac{2}{3 g_{m}} \cdot 32
\end{aligned}
$$

Thus, the cancellation noise power is

$$
\frac{P_{n 1}}{P_{n 2}}=108.1=20.3 \mathrm{~dB} .
$$

\section{SIMULATION AND EXPERIMENT RESULT}

Detailed circuits are shown in Figure 4, compared to the AC coupling model (Figure 2), the improved circuit is DC coupling amplifier with low frequency feedback loop. The multistage amplifier gain is set 100db in the example, the excitation signal of Manchester coded data with $5 \mathrm{mV}$ DC offset are applied to both circuits.

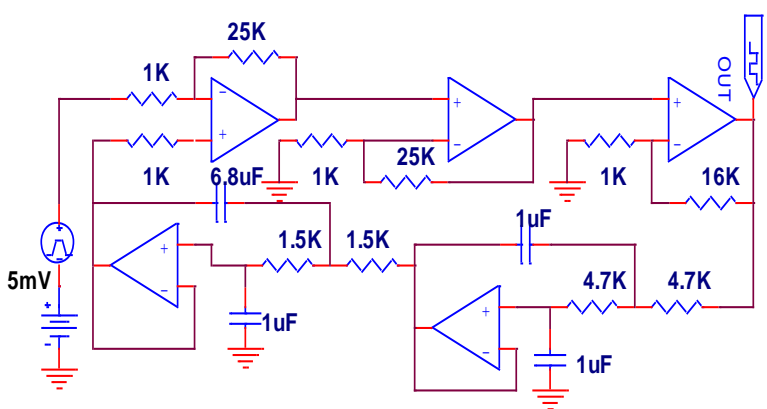

FIGURE IV. IMPROVED DC COUPLING AMPLIFIER CIRCUIT WITH LOW FREQUENCY SERVO LOOP.

The simulation results (Figure 5) show that both circuit can remove the DC offset, but the AC coupling amplifier cannot adapt various data rate, the distortion is serious in low and high data rate because of fixed AC constant, while DC coupling amplifier with low frequency servo loop can keep the signal integrity, no matter how much speed, the signal has no significant distortion. 


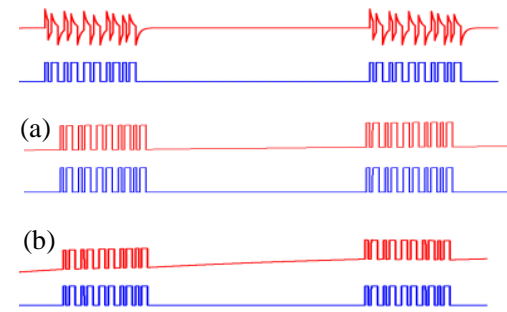

FIGURE V. (A) (B) (C) SHOW THE COMPARISON RESULTS OF AC COUPLING AND IMPROVED DC COUPLING AMPLIFIER IN 16KBPS,160KBPS AND 320KBPS DATA RATE.

This circuit is applied in UHF RFID system, and the test result is shown in Figure 6, the wave of baseband signal reflected from the tag can maintain very well in different data rate.
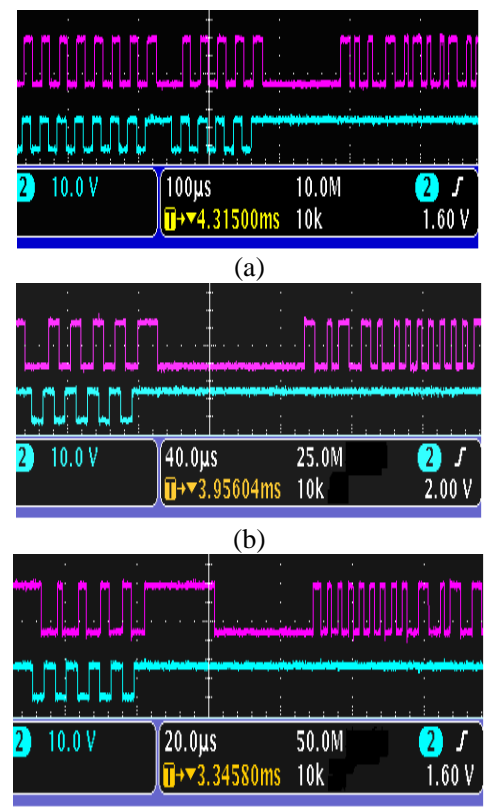

(c)

FIGURE VI. MEASURED WAVEFORM OF THE READER AND TAG, PINK COLOUR(TOP) IS TAG REFLECTED SIGNAL, AND BLUE COLOUR (BOTTOM)IS READER COMMAND. (A) (B)AND(C) IS 40 KBPS,80KBPS AND160KBPS DATA RATE.

\section{CONCLUSION}

The improved high-gain amplifier with low frequency servo loop circuit overcome the circuit saturation and solves the signal distortion problem. The experiments turn out the circuit can be applied in various signal transmission speed and promote the efficiency of the subsequent anti-collision algorithm in UHF RFID system. Beside, the circuit can also be used in other DCR system.

\section{ACKNOWLEDGMENT}

This work was supported by "Colleges and universities in Jiangsu Province plans to graduate research and innovation projects CXZZ13_0472"

\section{REFERENCES}

[1] B. Razavi, "Design Considerations for Direct-Conversion Receivers," in IEEE Transactions on Circuits and Systems- ii Analog and Digital Signal Processing, Vol. 44, No.6, 1997, pp.428-435.

[2] W. Namgoong and T. H. Meng, "Direct-Conversion RF Receiver Design," in IEEE Trans. on Comm., Vol. 49, No.3, 2001, pp. 518-529.

[3] Gilsung Byeon,"A New DC Offset Removal Algorithm Using an Iterative Method for Real-Time Simulation" IEEE TRANSACTIONS ON POWER DELIVERY, VOL. 26, NO. 4, OCTOBER 2011.

[4] L. Yu and W. M. Snelgrove, "A novel adaptive mismatch cancellation system for quadrature IF radio receivers," IEEE Trans. Circuits Syst. II, vol. 46, pp. 789-801, Jun 1999.

[5] Mingzheng Cao, Communications Systems, 2004. ICCS 2004. The Ninth International Conference on Date of Conference 7-7 Sept. 2004.

[6] H. Yoshida, H. Tsurumi and Y. Suzuki, "DC Offset Canceller in a Direct Conversion Receiver for QPSK Signal Reception," in the Ninth IEEE International Symposium on Personal. Indoor and Mobile Radio Comm. Vol. 3, 1998, pp. 1314-1318.

[7] EPC Radio-Frequency Identification Protocols Class-1 Gen-2 UHF RFID Protocol for Communications at $860 \mathrm{MHz}-960 \mathrm{MHz}$. 